\title{
POLVOS DE ESPECIAS AROMÁTICAS PARA EL CONTROL DEL GORGOJO DEL MAÍZ, Sitophilus zeamais MOTSCHULSKY, EN TRIGO ALMACENADO
}

\author{
Spices powders for the control of maize weevil, Sitophilus zeamais Motschulsky, \\ in stored wheat
}

\author{
Yessica Salvadores U., ${ }^{1}$ Gonzalo Silva A., ${ }^{1}$ Maritza Tapia V. ${ }^{1}$, y Ruperto Hepp G. ${ }^{1}$
}

\begin{abstract}
A B S T R A C T
The maize weevil, Sitophilus zeamais Motschulsky, is considered one of the most important pests of stored grains. Plant powders from nine seasoning spices were tested in the laboratory to control $S$. zeamais at $0.5,1$, 2 and $4 \%(\mathrm{w} / \mathrm{w})$. The variables evaluated were mortality and emergence (F1) of adult insects, grain weight loss and grain germination. The experimental design was completely randomized, with four replicates, and the group of treatments was repeated three times. The highest mortality percentages were obtained with Piper nigrum L. at 1\% (83.4\%), 2\% $(97.6 \%)$ and $4 \%(100 \%)$. The lowest adult insect emergence was obtained with the same treatments more Capsicum annuum var. longum Sendtn., Cinnamomum zeylanicum Blume and Pimpinella anisum L. at 4\% (w/w). No significant differences were recorded as regard grain weight loss and germination. All the plant powders tested had a repellent effect on Sitophilus zeamais and no fumigant effect was recorded.
\end{abstract}

Key words: stored grains, spices, condimentary species, Triticum aestivum.

\section{R E S U M E N}

El gorgojo del maíz Sitophilus zeamais Motschulsky, es considerado una de las plagas más importantes de productos almacenados. Se evaluaron, en laboratorio, polvos vegetales provenientes de nueve especias condimentarias para el control de $S$. zeamais en concentraciones de 0,$5 ; 1 ; 2$ y $4 \%$. Las variables evaluadas fueron mortalidad y emergencia (F1) de adultos y pérdida de peso y germinación del grano. También se evaluó el efecto repelente y fumigante a las concentraciones de 0,$5 ; 1$ y $2 \%$. El diseño experimental fue completamente al azar, los tratamientos tuvieron cuatro repeticiones y el grupo de tratamientos fue repetido tres veces. Las mayores mortalidades se obtuvieron con Piper nigrum L. a $1 \%$ $(83,4 \%), 2 \%(97,6 \%)$ y $4 \%(100 \%)$. Las menores emergencias de insectos adultos se obtuvieron en los mismos tratamientos más Capsicum annuum var. Longum Sendtn., Cinnamomun zeylanicum Blime y Pimpinella anisum $\mathrm{L}$. al $4 \%$ (p/p). La pérdida de peso y germinación de granos no registraron diferencia significativa. Todos los polvos vegetales fueron repelentes para Sitophilus zeamais y ninguno tuvo efecto fumigante.

Palabras clave: granos almacenados, sustancias aromáticas, especies condimentarias, Triticum aestivum.

\footnotetext{
${ }^{1}$ Universidad de Concepción, Facultad de Agronomía, Av. Vicente Méndez 595, Casilla 537, Chillán, Chile. E-mail: gosilva@udec.cl_*Autor para correspondencia.

Recibido: 10 de febrero de $2006 . \quad$ Aceptado: 4 de julio de 2006.
} 


\section{INTRODUCCIÓN}

La pérdida de granos en almacenaje es el principal problema en postcosecha que enfrenta el agricultor (Larraín, 1994). Entre todos los agentes perjudiciales, los insectos son los causantes de las mayores pérdidas (White, 1995), siendo las especies más importantes Sitophilus oryzae L., S. granarius L. y $S$. zeamais Motschulsky (Rees, 1996). Larraín (1994) señala que cerca del $10 \%$ de los granos de cereales pueden ser infestados por $S$. zeamais en el momento de la cosecha, y si la infestación continúa en el almacenaje, alrededor del 30 al 50\% de los granos pueden estar dañados al cabo de seis meses. El método químico es el más utilizado para proteger los granos almacenados del ataque de los insectos (White y Leesch, 1996). Sin embargo, los problemas causados por el mal uso de los insecticidas sintéticos han obligado a buscar nuevas alternativas de control, como es el uso de sustancias derivadas del metabolismo secundario de las plantas (Mareggiani, 2001).

El uso más sencillo de estos compuestos en la protección de granos almacenados es como polvos. Las plantas se secan, luego se muelen, y se mezclan con el grano, lo que modifica el ecosistema de las plagas presentes en los granos almacenados (Weaver y Subramanyam, 2000). Los efectos más significativos en el comportamiento de los insectos están relacionados con la selección del hospedero para alimentación y oviposición, y en cuanto a la alteración del metabolismo las consecuencias más importantes son aquellas relacionadas con la duración del ciclo del insecto, fecundidad y sobrevivencia (Rodríguez y Lagunes, 1992). La mayoría de las especies vegetales utilizadas como insecticidas no eliminan al insecto por intoxicación, sino que generalmente inhiben su desarrollo normal, al actuar como repelentes o disuasivos de la alimentación u oviposición, lo cual hace que muchas veces se sobredimensionen sus efectos protectores (Silva et al., 2002).

El objetivo de la presente investigación fue evaluar las propiedades insecticidas/insectistáticas de nueve especies vegetales condimentarias para el control de $S$. zeamais en laboratorio.

\section{MATERIALES Y MÉTODOS}

\section{Tratamientos}

El material vegetal usado se compró en el mercado de frutas y hortalizas de Chillán, VIII Región, Chile (Cuadro 1). Este material se encontraba deshidratado y molido, por lo que no fue posible establecer la edad y procedencia del mismo. Con el objeto de homogeneizar la granulometría de los polvos, se molieron nuevamente con un molino eléctrico para café y se tamizaron con un tamiz de 250 micrones (DUAL Manufacturing Co., Chicago, Illinois, USA). Se evaluaron 37 tratamientos incluyendo un testigo absoluto sin polvo vegetal. Cada especie se evaluó en concentraciones de 0,5 ; $1 ; 2$ y $4 \%$ de polvo vegetal en $20 \mathrm{~g}$ de trigo (Triticum aestivum L.) (p/p) con cuatro repeticiones. Se utilizaron adultos del gorgojo del maíz, S. zeamais Motschulsky, los cuales se reprodujeron bajo condiciones controladas de temperatura $\left(30 \pm 2^{\circ} \mathrm{C}\right)$ y oscuridad total en una cámara bioclimática. Los insectos utilizados eran adultos de entre 1 y 10 días de edad, y se diferenciaron por sexo de acuerdo al criterio propuesto por Halstead (1963).

Cuadro 1. Especies vegetales aromáticas evaluadas para el control de Sitophilus zeamais en laboratorio. Table 1. Plant seasoning species evaluated for Sitophilus zeamais control under laboratory conditions.

\begin{tabular}{lll}
\hline Nombre científico & Familia & Nombre común \\
\hline Capsicum annuum Sendtn. var. grossum & Solanaceae & Ají de color \\
Capsicum annuum Sendtn. var. longum & Solanaceae & Ají cacho de cabra \\
Cinnamomum zeylanicum Blume & Lauraceae & Canela \\
Cuminum cyminum L. & Apiaceae & Comino \\
Myristica fragrans Houtt. & Myristicaceae & Nuez moscada \\
Origanum vulgare L. & Lamiaceae & Orégano \\
Pimpinella anisum L. & Apiaceae & Anís \\
Piper nigrum L. & Piperaceae & Pimienta negra \\
Syzygium aromaticum (L.) & Myristicaceae & Clavo de olor \\
& &
\end{tabular}




\section{Metodología y evaluación}

La metodología utilizada fue la propuesta por Tavares (2002). En placas Petri de $6 \mathrm{~cm}$ de diámetro se mezclaron $20 \mathrm{~g}$ de trigo cv. Nobo $(14 \%$ de humedad) con los diferentes polvos vegetales a las respectivas concentraciones. Una vez realizada la mezcla, cada placa se infestó con 10 parejas de insectos, y para estimar la toxicidad de cada uno de los tratamientos se midieron las siguientes variables:

Porcentaje de mortalidad de insectos adultos. Se evaluó a los 15 días de realizada la infestación, contabilizando los insectos adultos vivos y muertos de cada tratamiento, que se retiraron de las placas en forma definitiva. El porcentaje de mortalidad obtenido se corrigió con la fórmula de Abbott (Abbott, 1925).

Porcentaje de emergencia de insectos adultos (F1). Se evaluó a los 55 días de realizada la infestación y la emergencia del testigo se consideró como $100 \%$.

Porcentaje de pérdida de peso y germinación de los granos. Se evaluó a los 55 días de realizada la infestación. Para su estimación se utilizó la diferencia de peso entre los $20 \mathrm{~g}$ iniciales de cada placa y el peso al final de la evaluación. La germinación de los granos de trigo se evaluó en aquellos tratamientos que obtuvieron una mortalidad superior al $80 \%$. De estos tratamientos se escogieron 40 semillas al azar, las cuales se hicieron germinar a temperatura ambiente en placas Petri acondicionadas con papel absorbente humedecido; siete días después se contabilizó el número de semillas germinadas, comparándolas con el testigo que se consideró como $100 \%$.

Repelencia. La metodología utilizada para medir la repelencia fue la propuesta por Tavares (2002). Se utilizó una estructura formada por cinco placas Petri plásticas, estando una placa central conectada con las demás por tubos plásticos dispuestos diagonalmente en un ángulo de $45^{\circ}$. Los tratamientos con polvos vegetales en concentraciones de 0,$5 ; 1$ y $2 \%$ en $20 \mathrm{~g}$ de trigo $(\mathrm{p} / \mathrm{p})$ y el testigo sin polvo vegetal, se distribuyeron en dos placas simétricamente opuestas. En la placa central se liberaron 50 individuos adultos de $S$. zeamais y luego de 24 h se contabilizó el número de individuos presentes en cada placa. Cada tratamiento tuvo cinco repeticio- nes y con estos valores se calculó un índice de repelencia (IR) según lo descrito por Mazzonetto (2002), donde el polvo se clasifica como neutro si el índice es igual a 1 , atrayente si es mayor a $1 \mathrm{y}$ repelente si es menor a 1 .

$$
\mathrm{IR}=\frac{2 G}{(G+P)}
$$

donde G: porcentaje de insectos en el tratamiento, y P: porcentaje de insectos en el testigo.

Efecto fumigante. Para evaluar el efecto fumigante se utilizó la metodología propuesta por Tavares (2002). En frascos de plástico de $100 \mathrm{~mL}$ se dispuso en el fondo y centrado un tubo de PVC en cuyo interior se colocaron los polvos vegetales a las concentraciones de 0,$5 ; 1$ y $2 \%(\mathrm{p} / \mathrm{p})$, el cual fue cubierto por un trozo de tul fino. Alrededor de este tubo se colocaron $40 \mathrm{~g}$ de trigo, el cual se infestó con 20 individuos de $S$. zeamais. El tul tuvo como objetivo impedir el contacto de los insectos con los polvos, pero permitir que se desprendieran al medio los compuestos volátiles presentes en ellos y que podrían resultar tóxicos para los insectos al ser inhalados. Los frascos se mantuvieron en una cámara bioclimática a $25 \pm 2^{\circ} \mathrm{C}$ y las mediciones de mortalidad por inhalación se realizaron a los cinco días de realizada la infestación, contabilizado los adultos muertos y vivos.

Se usó un diseño experimental completamente al azar. Los datos obtenidos se transformaron con la fórmula arcoseno $\sqrt{x / 100}$ y se sometieron a un análisis de varianza utilizando el software Statistical Analysis System (SAS Institute, 1998) y se realizó un test de comparación de medias mediante una prueba de Tukey con un nivel de significancia del $95 \%$.

\section{RESULTADOS Y DISCUSIÓN}

\author{
Mortalidad \\ De acuerdo al criterio señalado por Lagunes (1994), \\ que califica como prometedor al polvo vegetal que \\ supere el $50 \%$ de mortalidad, se puede señalar que \\ 23 de los 36 tratamientos evaluados cumplen con \\ este criterio. Los mejores resultados se obtuvieron \\ con el polvo de Piper nigrum L. (pimienta negra), \\ el cual a partir de la concentración $1 \%$ provocó una \\ mortalidad de insectos superior al $80 \%$. Además, \\ Cinnamomum zeylanicum (canela), Cuminum cymi-
}


num L. (comino), Myristica fragans Houtt. (nuez moscada) y Origanum vulgare L. (orégano) registraron valores entre 80 y $100 \%$ de mortalidad de adultos de $S$. zeamais a la concentración de $4 \%$ (Cuadro 2). Los granos tratados con polvo de Pimpinella anisum L. y C. cyminum al $1 \%$ registraron un 30,2 y $52,2 \%$ de mortalidad de insectos, valor mayor al de Páez (1987), quien obtuvo un 3,1 y $1,8 \%$ de mortalidad, respectivamente, con los mismos polvos y concentración aunque no indica el origen y características del material vegetal. Los resultados exhibidos por $P$. nigrum concuerdan con

Cuadro 2. Efecto de polvos de vegetales aromáticos a diferentes concentraciones sobre la mortalidad y emergencia de insectos y porcentaje de pérdida de peso de los granos de trigo infestados por Sitophilus zeamais.

Table 2. Effect of seasoning plants powder at different concentrations on insect mortality and emergence and wheat grain weight loss infested by Sitophilus zeamais.

\begin{tabular}{|c|c|c|c|c|}
\hline Tratamientos & $\begin{array}{c}\text { Concentración } \\
(\%)\end{array}$ & $\begin{array}{c}\text { Mortalidad } \\
(\%)\end{array}$ & $\begin{array}{c}\text { Emergencia } \\
(\%)\end{array}$ & $\begin{array}{c}\text { Pérdida de peso }{ }^{1} \\
(\%)\end{array}$ \\
\hline \multirow[t]{4}{*}{ Capsicum annuum var. grossum } & 0,5 & 12,3 abcde & 48,7 ghi & 6,1 \\
\hline & 1 & 28,3 abcde & 57,2 cdefg & 5,8 \\
\hline & 2 & 56,1 abcde & $34,3 \mathrm{jklm}$ & 4,7 \\
\hline & 4 & 71,4 abcde & $35,9 \mathrm{jkl}$ & 4,2 \\
\hline \multirow[t]{4}{*}{ Capsicum annuum var. longum } & 0,5 & 26,6 abcde & $78,7 \mathrm{~b}$ & 7,3 \\
\hline & 1 & 40,6 abcde & 56,0 defg & 5,2 \\
\hline & 2 & 50,9 abcde & 30,6 klmno & 3,9 \\
\hline & 4 & 66,7 bcde & $10,8 \mathrm{rs}$ & 3,3 \\
\hline \multirow[t]{4}{*}{ Cinnamomum zeylanicum } & 0,5 & $29,0 \mathrm{cde}$ & 67,9 bc & 6,9 \\
\hline & 1 & 43,8 cde & $35,7 \mathrm{jkl}$ & 5,0 \\
\hline & 2 & $61,6 \mathrm{de}$ & 23,9 mnop & 3,5 \\
\hline & 4 & 80,0 e & $7,9 \mathrm{~s}$ & 2,7 \\
\hline \multirow[t]{4}{*}{ Cuminum cyminum } & 0,5 & $36,7 \mathrm{abc}$ & $52,4 \mathrm{fgh}$ & 4,9 \\
\hline & 1 & $52,2 \mathrm{abcd}$ & 49,7 ghi & 5,0 \\
\hline & 2 & $69,1 \mathrm{abcd}$ & 42,0 hij & 4,2 \\
\hline & 4 & 83,1 abcde & $36,0 \mathrm{jkl}$ & 4,6 \\
\hline \multirow[t]{4}{*}{ Myristica fragans } & 0,5 & 41,4 abcde & $78,8 \mathrm{~b}$ & 6,6 \\
\hline & 1 & 57,4 abcde & 56,9 defg & 5,6 \\
\hline & 2 & 67,2 abcde & $48,1 \mathrm{ghi}$ & 4,8 \\
\hline & 4 & 84,8 abcde & $37,1 \mathrm{jkl}$ & 4,0 \\
\hline \multirow[t]{4}{*}{ Origanum vulgare } & 0,5 & $41,7 \mathrm{a}$ & $39,6 \mathrm{ijk}$ & 4,6 \\
\hline & 1 & $63,0 \mathrm{ab}$ & $39,0 \mathrm{ijk}$ & 4,7 \\
\hline & 2 & $69,5 \mathrm{abc}$ & 21,3 opqr & 3,2 \\
\hline & 4 & $81,1 \mathrm{abc}$ & 21,8 nopq & 3,2 \\
\hline \multirow[t]{4}{*}{ Pimpinella anisum } & 0,5 & 20,2 abcde & $66,0 \mathrm{~cd}$ & 6,3 \\
\hline & 1 & 30,2 abcde & $60,8 \mathrm{cdef}$ & 6,2 \\
\hline & 2 & 45,7 abcde & $53,2 \mathrm{efg}$ & 6,1 \\
\hline & 4 & 62,3 abcde & $19,5 \mathrm{pqr}$ & 4,2 \\
\hline \multirow[t]{4}{*}{ Piper nigrum } & 0,5 & $65,6 \mathrm{cde}$ & 32,3 jklmn & 4,0 \\
\hline & 1 & 83,4 cde & 26,7 lmnop & 3,6 \\
\hline & 2 & $97,6 \mathrm{cde}$ & $11,7 \mathrm{qrs}$ & 2,9 \\
\hline & 4 & $100,00 \mathrm{cde}$ & $3,4 \mathrm{~s}$ & 2,6 \\
\hline \multirow[t]{4}{*}{ Syzygium aromaticum } & 0,5 & 29,1 abcde & 63,4 cde & 6,4 \\
\hline & 1 & 39,3 abcde & 39,7 ijk & 4,9 \\
\hline & 2 & 54,0 abcde & $39,1 \mathrm{ijk}$ & 4,9 \\
\hline & 4 & 66,1 abcde & 24,4 mnop & 3,9 \\
\hline Testigo & & --- & $100,0 \mathrm{a}$ & 7,7 \\
\hline CV (\%) & & 34,40 & 8,06 & 63,86 \\
\hline
\end{tabular}

Tratamientos con igual letra en la columna no difieren estadísticamente según prueba de Tukey ( $\mathrm{p} \leq 0,05)$.

1 No hubo diferencias significativas; CV: coeficiente de variación. 
De Assis et al. (1999), quienes encontraron que el extracto de esta planta es altamente tóxico para Sitophilus spp., y Awoyinka et al. (2006) que atribuyen esta toxicidad a que los frutos de esta planta tienen una concentración cercana al $4 \%$ de alcaloides como piperina, piperetina y tricostacina entre otros. Por otro lado, los resultados más bajos se obtuvieron con Capsicum annuum var. grossum y con P. anisum con un 12 y $20 \%$, respectivamente, a una concentración de $0,5 \%$. Cabe destacar que los bajos resultados obtenidos con $C$. annuum son similares a los de Procopio et al. (2003) con polvo de hojas y frutos de Capsicum frutescens L. a una concentración de $3 \%$, quienes obtuvieron una mortalidad de S. zeamais de 4,17 y $9,17 \%$, respectivamente.

\section{Emergencia de insectos adultos (F1)}

La menor emergencia $(3,4 \%)$ de la F1 se registró con los polvos de $P$. nigrum al $4 \%$ aunque sin diferencias estadísticas con $C$. zeylanicum y $C$. annuum var. longum a la misma concentración y $P$. nigrum al $2 \%$ (Cuadro 2). Sin embargo, se debe señalar que todos los tratamientos presentaron valores menores y estadísticamente diferentes al testigo. Los resultados de $P$. nigrum concuerdan con los registrados por Aslam et al. (2002), quienes al evaluar esta especia en granos de garbanzo (Cicer arietinum L.) contra el gorgojo sureño del frejol, Callosobruchus chinensis L. (Coleoptera: Bruchidae), registraron un menor número de adultos. Estos autores atribuyen esta disminución a los componentes activos de esta especie, como la piperina, los cuales podrían alterar el comportamiento fisiológico del insecto en la oviposición, inhibiendo el crecimiento o directamente en la mortalidad de adultos. Cabe destacar que algunas especies como C. cyminum, $C$. zeylanicum y $S$. aromaticum, incluso en concentraciones bajas como $1 \%(\mathrm{p} / \mathrm{p})$, a pesar de presentar una baja mortalidad mostraron una emergencia de insectos adultos menor al 50\% en comparación al testigo. De hecho, si se considera el criterio de Lagunes (1994) que clasifica como prometedores a aquellos polvos que reducen en un $50 \%$ la $\mathrm{F} 1$, podemos señalar que 25 de los 36 tratamientos evaluados se pueden clasificar como prometedores. Esto podría indicar que estos polvos presentan algún tipo de efecto disuasivo de la oviposición, es decir modifican el comportamiento reproductivo. Aunque en el caso de C. cyminum los resultados contradicen a Páez (1987), en cuyo caso al tratar granos de maíz infestados con $S$. zeamais la emergencia en $C$. cyminum superó en un $9,5 \%$ al testigo que era considerado como el $100 \%$.

\section{Porcentaje de pérdida de peso de los granos}

En el Cuadro 2 se observa que la pérdida de peso de los granos fue baja, registrándose valores que fluctúan entre 2,6 y 7,3\%, no existiendo diferencia estadística significativa para ninguno de los polvos vegetales con respecto al testigo. Los datos obtenidos concuerdan con los descritos por Aslam et al. (2002), quienes al tratar granos de garbanzo con $S$. aromaticum y P. nigrum contra Callosobruchus chinensis obtuvieron una baja pérdida de peso, la cual se podría deber a la mortalidad temprana de los insectos con una consecuente menor oviposición por grano.

\section{Germinación}

Esta prueba se realizó sólo en aquellos tratamientos que sobrepasaron el $80 \%$ de mortalidad de adultos. Éstos fueron los polvos de $C$. zeylanicum, $C$. cyminum, $M$. fragans y $O$. vulgare al $4 \%(\mathrm{p} / \mathrm{p})$ y $P$. nigrum al 1; 2 y $4 \%(\mathrm{p} / \mathrm{p})$. El Cuadro 3 muestra los valores obtenidos en cada uno de los tratamientos evaluados pudiéndose constatar que el polvo de las especias no afectó significativamente el poder germinativo de los granos, ya que no se registró diferencia estadística entre los tratamientos evalua-

Cuadro 3. Porcentaje de germinación de granos de trigo mezclados con polvos de Cinnamomum zeylanicum, Cuminum cyminum, Myristica fragrans, Origanum vulgare y Piper nigrum a las concentraciones que se indica.

Table 3. Percentage of wheat grain germination mixed with powders of Cinnamomum zeylanicum, Cuminum cyminum, Myristica fragrans, Origanum vulgare and Piper nigrum at the indicates concentrations.

\begin{tabular}{lcc}
\hline Especie evaluada & $\begin{array}{c}\text { Concentración } \\
\text { (\%) }\end{array}$ & $\begin{array}{c}\text { Germinación } \\
\text { (\%) }\end{array}$ \\
\hline Cinnamomum zeylanicum & 4 & 78,3 \\
Cuminum cyminum & 4 & 84,2 \\
Myristica fragrans & 4 & 93,3 \\
Origanum vulgare & 4 & 93,3 \\
Piper nigrum & 1 & 88,3 \\
& 2 & 85,0 \\
Testigo & 4 & 76,7 \\
CV (\%) & & 100 \\
& & 10,84 \\
\hline
\end{tabular}

*Tratamientos con igual letra en la columna no difieren estadísticamente según prueba de Tukey $(\mathrm{p} \leq 0,05)$.

1 No hubo diferencias significativas; CV: coeficiente de variación. 
dos y el testigo. Sin embargo, aunque no existen diferencias estadísticas, los datos muestran diferencias de hasta un $30 \%$ con el testigo, lo cual podría deberse a que el trigo utilizado en las evaluaciones estaba destinado a alimentación animal y no para semilla. Estos datos coinciden con los resultados de Sighamony et al. (1986), quienes obtuvieron un $100 \%$ de germinación en granos de trigo tratados con extractos de P. nigrum usados para el control de $S$. oryzae y Rhyzopertha dominica L., concluyendo que el extracto de esta planta no afectaba la viabilidad de las semillas. Ahmed y Ahamad (1992) probaron la eficacia de varias plantas con propiedades medicinales y de uso culinario contra C. chi- nensis, concluyendo que las especies vegetales, aparte de no afectar la germinación, no presentan toxicidad para mamíferos y pueden, por lo tanto, ser incorporadas en forma segura para la protección prolongada contra insectos de granos almacenados en concentraciones de hasta $3 \%$.

\section{Repelencia}

Los resultados obtenidos indican que todos los polvos vegetales evaluados tuvieron efecto repelente (Cuadro 4). S. aromaticum al $2 \%$ se destacó por su mayor efecto repelente ( $I R=0,32$ ), mientras que con $O$. vulgare se obtuvo el valor más cercano a 1 $(\mathrm{IR}=0,83)$. Los datos registrados coinciden con

Cuadro 4. Índice de repelencia y mortalidad de Sitophilus zeamais al evaluar el efecto fumigante de polvos de vegetales aromáticos en granos de trigo tratados a las concentraciones que se indica.

Table 4. Repelence index and mortality of Sitophilus zeamais at the evaluation of fummigant effect of aromatic plants powders in wheat grains treated at the indicated concentrations.

\begin{tabular}{|c|c|c|c|}
\hline Tratamiento & $\begin{array}{c}\text { Concentración } \\
(\%)\end{array}$ & $\begin{array}{l}\text { Índice de repelencia } \\
\text { (IR) }\end{array}$ & $\begin{array}{c}\text { Mortalidad } \\
\text { (\%) }\end{array}$ \\
\hline \multirow[t]{3}{*}{ Capsicum annuum var. grossum } & 0,5 & 0,59 & $2,2 \mathrm{bc}$ \\
\hline & 1 & 0,63 & $3,9 \mathrm{bc}$ \\
\hline & 2 & 0,60 & $7,2 \mathrm{bc}$ \\
\hline \multirow[t]{3}{*}{ Capsicum annuum var. longum } & 0,5 & 0,76 & $1,7 \mathrm{bc}$ \\
\hline & 1 & 0,75 & $4,4 \mathrm{bc}$ \\
\hline & 2 & 0,57 & $7,8 \mathrm{bc}$ \\
\hline \multirow[t]{3}{*}{ Cinnamomum zeylanicum } & 0,5 & 0,75 & $8,3 \mathrm{c}$ \\
\hline & 1 & 0,71 & $12,8 \mathrm{c}$ \\
\hline & 2 & 0,68 & $20,6 \mathrm{c}$ \\
\hline \multirow[t]{3}{*}{ Cuminum cyminum } & 0,5 & 0,79 & $10,0 \mathrm{abc}$ \\
\hline & 1 & 0,52 & $18,9 \mathrm{abc}$ \\
\hline & 2 & 0,61 & $27,2 \mathrm{abc}$ \\
\hline \multirow[t]{3}{*}{ Myristica fragans } & 0,5 & 0,63 & $2,2 \mathrm{bc}$ \\
\hline & 1 & 0,50 & $3,9 \mathrm{bc}$ \\
\hline & 2 & 0,65 & $6,7 \mathrm{bc}$ \\
\hline \multirow[t]{3}{*}{ Origanum vulgare } & 0,5 & 0,83 & $6,1 \mathrm{a}$ \\
\hline & 1 & 0,76 & $7,8 \mathrm{ab}$ \\
\hline & 2 & 0,66 & $10,0 \mathrm{abc}$ \\
\hline \multirow[t]{3}{*}{ Pimpinella anisum } & 0,5 & 0,78 & $6,7 \mathrm{bc}$ \\
\hline & 1 & 0,74 & $12,8 \mathrm{bc}$ \\
\hline & 2 & 0,62 & $18,9 \mathrm{bc}$ \\
\hline \multirow[t]{3}{*}{ Piper nigrum } & 0,5 & 0,75 & $1,1 \mathrm{bc}$ \\
\hline & 1 & 0,75 & $2,8 \mathrm{bc}$ \\
\hline & 2 & 0,77 & $4,4 \mathrm{bc}$ \\
\hline \multirow[t]{3}{*}{ Syzygium aromaticum } & 0,5 & 0,70 & $4,4 \mathrm{bc}$ \\
\hline & 1 & 0,62 & $8,3 \mathrm{bc}$ \\
\hline & 2 & 0,32 & $16,7 \mathrm{bc}$ \\
\hline CV (\%) & - & - & 59,52 \\
\hline
\end{tabular}

Tratamientos con igual letra en la columna no difieren estadísticamente según prueba de Tukey ( $\mathrm{p} \leq 0,05)$.

CV: coeficiente de variación.

IR: 1 = neutro; IR $>1$ atrayente; IR $<1$ repelente. 
los obtenidos por Sighamony et al. (1984), quienes evaluaron el efecto repelente de $S$. aromaticum y $P$. nigrum contra adultos de Tribolium castaneum, concluyendo que el extracto de $P$. nigrum a la concentración más alta evaluada tenía un mayor efecto repelente que a su concentración más baja. Si bien los valores obtenidos por Sighamony et al. (1984) no se pueden comparar completamente con los datos registrados en la presente investigación, debido a que las especies vegetales fueron utilizadas en forma de extractos y no de polvo, permiten reafirmar el hecho que ambas especies presentan efecto repelente.

\section{Efecto fumigante}

Del total de polvos evaluados, ninguno mostró tener efecto fumigante cercano al $100 \%$. De hecho, los valores de mortalidad obtenidos fueron menores al 30\% (Cuadro 4). La mayor mortalidad de insectos se registró con los granos tratados con polvos de $C$. cyminum al $2 \%$ con una mortalidad de $27,2 \%$, mien- tras que el menor registro fue para P. nigrum al 0,5\% con un $1,1 \%$. Estos resultados, por lo tanto, permiten inferir que los polvos evaluados sólo tienen efecto insecticida de contacto y no gaseoso.

\section{CONCLUSIONES}

De las especies vegetales evaluadas, Cinnamomum zeylanicum Blume., Cuminum cyminum L., Myristica fragans Houtt., Origanum vulgare L. en concentraciones de $4 \%(\mathrm{p} / \mathrm{p})$ y Piper nigrum $\mathrm{L}$. en concentraciones de 1,2 y $4 \%$ presentan acción insecticida de contacto igual o superior al $80 \%$ contra $S$. zeamais.

La presencia de polvos vegetales de las especias evaluadas no afecta en forma significativa la germinación de los granos de trigo.

Todas las especias evaluadas son repelentes para Sitophilus zeamais en laboratorio.

\section{LITERATURA CITADA}

Abbott, W., 1925. A method for computing the effectiveness of an insecticide. J. Econ. Entomol. 18:265-267.

Ahmed, S.M., and A. Ahamad. 1992. Efficacy of some indigenous plants as pulse protectants against Callosobruchus chinensis L. infestation. Int. Pest Control 34:54-56.

Aslam, M., K. Ali Khan, and M. Bajwa. 2002. Potency of some spices against Callosobruchus chinensis Linnaeus. Online J. Biol. Sci. 2: 449-452.

Awoyinka,O.A., I.O., Oyewole, B.M. W. Amos and O.F. Onasoga. 2006. Comparative pesticidal activity of dichloromethane extracts of Piper nigrum against Sitophilus zeamais and Callosobruchus maculatus. African Journal of Biotechnology 5:2446-2449.

De Assis, C.F., A. Costa y J. Palmeira. 1999. Avaliação de extratos vegetais e métodos de aplicação no controle de Sitophilus spp. Revista Brasileira de Produtos Agroindustriais 1:13-20.

Halstead, D.G.H. 1963. External sex differences in storedproducts coleoptera. Bull. Entomol. Res. 54:119-134.

Lagunes, A. 1994. Extractos, polvos vegetales y minerales para el combate de plagas del maíz y del frijol en la agricultura de subsistencia. $35 \mathrm{p}$. Memoria. Colegio de Postgraduados en Ciencias Agrícolas, Montecillo, Texcoco, México.
Larraín, P. 1994. Manejo integrado de plagas en granos almacenados. Investigación y Progreso Agropecuario La Platina $\mathrm{N}^{\circ}$ 81. p. 10-16.

Mareggiani, G. 2001. Manejo de insectos plaga mediante sustancias semiquímicas de origen vegetal. Manejo Integrado de Plagas 60:22-30.

Mazzonetto, F. 2002. Efeito de genótipos de feijoeiro e de pós origem vegetal sobre Zabrotes subfasciatus (Boh.) e Acanthoscelides obtectus (Say) (Col. Bruchidae). 134 p. Tesis Doctor en Ciencias. Universidad de Sao Paulo, Piracicaba, Sao Paulo, Brasil.

Páez, A. 1987. Uso de polvos vegetales e inertes minerales como una alternativa para el combate del gorgojo del maíz Sitophilus zeamais Motschulsky (Coleoptera: Curculionidae) en maíz almacenado. 108 p. Tesis Magister en Ciencias. Colegio de Postgraduados en Ciencias Agrícolas, Montecillo, Texcoco, México.

Procopio, S., J. Vendramim, J. Ribeiro, y J. Barbosa. 2003. Bioatividad de diversos pós de origem vegetal en relaçao a Sitophilus zeamais Mots. (Coleóptera: Curculionidae). Ciência e Agrotecnologia 6:12311236. 
Rees, P. 1996. Coleoptera. p. 1-40. In B. Subramanyam, and D. Hagstrum (eds.) Integrated management of insects in stored products. Marcel Dekker, New York, USA.

Rodríguez, C., y A. Lagunes. 1992. Plantas con propiedades insecticidas: resultados de pruebas experimentales en laboratorio, campo y granos almacenados. Agroproductividad No 1. p. 17-25.

SAS Institute. 1998. Introductory guide for personal computer. Release 6.03. 1028 p. SAS Institute, Cary, North Carolina, USA.

Sighamony, S., I. Anees, T. Chandrakala, and Z. Osmani. 1984. Natural products as repellents for Tribolium castaneum Herbst. Int. Pest Control 26:156-159.

Sighamony, S., I. Anees, T. Chandrakala, and Z. Osmani. 1986. Efficacy of certain indigenous plant products as grain protectants against Sitophilus oryzae (L.) and Rhyzopertha dominica (F.). J. Stored Prod. Res. 22(1):21-23.

Silva, G., A. Lagunes, J.C. Rodríguez, y D. Rodríguez. 2002. Insecticidas vegetales; una vieja y nueva alternativa en el manejo de plagas. Manejo Integrado de Plagas y Agroecología 66:4-12.
Tavares, M. 2002. Bioatividade da erva de Santa Maria Chenopodium ambrosioides L. (Chenopodiaceae) em relaVâo a Sitophilus zeamais Mots., 1855. (Col.: Curculionidae). 59 p. Tesis Magister en Ciencias. Universidad de Sao Paulo, Piracicaba, Sao Paulo, Brasil.

Weaver, D. and B. Subramanyam. 2000. Botanicals. p. 303-320. In B. Subramanyam, and D. Hagstrum (eds.) Alternatives to pesticides in stored product IPM. Kluwer Academic Press, Boston, USA.

White, N. 1995. Insects, mites and insecticides in stored grain ecosystems p. 123-168. In D. Jayas, N. White, and W. Muir (eds.) Stored grain ecosystems. Marcel Dekker, New York, USA.

White, N., and J. Leesch. 1996. Chemical control. p. 287330. In B. Subramanyam, and D. Hagstrum (eds.) Integrated management of insects in stored products. Marcel Dekker, New York, USA. 